\title{
Validation of stellar population and kinematical analysis of galaxies
}

\author{
M. Koleva ${ }^{1,2}$, N. Bavouzet ${ }^{3}$, I. Chilingarian ${ }^{2,4}$, and P. Prugniel ${ }^{2,3}$ \\ 1 Department of Astronomy, Sofia University, Bulgaria \\ 2 CRAL - Observatoire de Lyon, France \\ 3 GEPI - Observatoire de Paris, France \\ 4 Sternberg Astronomical Institute, Moscow, Russia
}

\begin{abstract}
Summary. 3D spectroscopy produces hundreds of spectra from which maps of the characteristics of stellar populations (age-metallicity) and internal kinematics of galaxies can be derived. We carried on simulations to assess the reliability of inversion methods and to define the requirements for future observations. We quantify the biases and show that to minimize the errors on the kinematics, age and metallicity (in a given observing time) the size of the spatial elements and the spectral dispersion should be chosen to obtain an instrumental velocity dispersion comparable to the physical dispersion.
\end{abstract}

Key words: kinematics, stellar populations of galaxies, error analysis

\section{Analyzing the data}

Recently it became possible to derive simultaneously internal kinematics and characteristics of the stellar population of galaxies [1].

We use a simple parametric procedure [2] to fit the moments of the lineof-sight velocity distribution (LOSVD): $\mathrm{v}, \sigma, \ldots$; and parametrized star formation history (SFH) containing either single stellar population (SSP) or several star bursts, using models computed with PEGASE.HR [3]. Before running the inversion procedure one needs to: (a) determine variations of the spectrograph's line-spread function along wavelength and over the field of the IFU; (b) inject this information into template spectra. We stress that the population parameters are constrained by the absorption lines and not by the shape of the continuum which may be affected by internal extinction or calibration uncertainties.

\section{Validation of the population pixel fitting}

The questions we address are:

1. Does the method return unbiased estimates of kinematics, age $(t)$ and metallicity $(Z)$ at any signal to noise ratio (SNR)?

2. What are the degeneracies between different parameters? 
3. What SNR and spectral resolution are required to get a given precision?

4. Are various models of stellar populations consistent?

Biases and degeneracy. We have performed extensive Monte-Carlo simulations to fit SSP or SFH containing two bursts with SNR ranging from 1 to $500 \mathrm{pix}^{-1}$ (at $\mathrm{R}=10000$; $\mathrm{pix}=0.2 \AA$ ). As long as the grid of models is fine enough for performing good interpolation, our method is not biased down to $\mathrm{SNR}=5 \mathrm{pix}^{-1}$. The main degeneracy is naturally between age and metallicity, but Fig 1 presents also the degeneracy between $\sigma$ and $Z$ : a discrepancy toward higher $Z$ (sharp lines) is compensated by higher $\sigma$. This latter degeneracy is the strongest coupling between the kinematical and population parameters. An error of 1 dex on the metallicity results in an error of about $25 \%$ on the velocity dispersion. This can introduce a significant systematics in the determination of the mass-to-light ratio of galaxies. This effect is considerably reduced if an additive continuum is included in the fit, but in this case constraints on the stellar populations are lost.

Relation between precision and SNR. The precision essentially depends on the total SNR integrated over the whole wavelength range (Fig 2): a lower SNR per pixel can be balanced by a larger number of pixels. When the wavelength range is shortened to exclude the blue region containing $\mathrm{H}_{\beta}$ line and bluer (but keeping the same total SNR), the precision on kinematics and metallicity is not seriously affected, but the precision on age becomes twice worse.
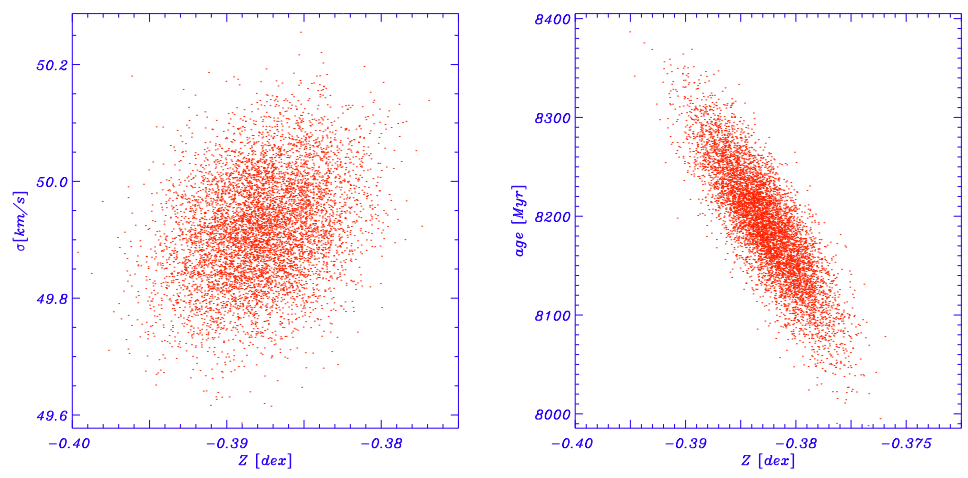

Fig. 1. Metallicity-velocity dispersion and Age-metallicity degeneracies. MonteCarlo simulations with PEGASE.HR spectra, $\mathrm{SNR}=100 \mathrm{pix}^{-1}, \sigma=50 \mathrm{~km} \mathrm{~s}^{-1}$

Relation between resolution and precision. The precision on kinematical and population parameters depends on $\left(\sigma^{2}+\sigma_{\text {ins }}^{2}\right)($ Fig 3$)$, where $\sigma_{\text {ins }}$ is the instrumental velocity dispersion. The resolution is an important parameter for the precise determination of the kinematics. But it has weaker influence over the errors on age and metallicity .(See the relations below.) When $\sigma<$ 
$0.4 \sigma_{\text {ins }}$, the different minimization strategies that we experimented generally become unstable to measure the internal kinematics.

\section{Conclusion}

The method based on the pixel fitting with PEGASE.HR templates can efficiently constrain kinematics and stellar population. We summarize below the relations between the errors on the population characteristics, the total signal to noise ratio, and the resolution (for the spectral range $4000 \AA-6800 \AA$ ):

$$
\begin{aligned}
e r r_{\mathrm{v}} & \approx 5 \times S N R^{-1} \times\left(\sigma^{2}+\sigma_{\text {ins }}^{2}\right)^{2 / 3} \\
\sigma \times e r r_{\sigma} & \approx 5 \times S N R^{-1} \times\left(\sigma^{2}+\sigma_{\text {ins }}^{2}\right)^{6 / 5} \\
e r r_{t} / t & \approx 54 \times S N R^{-1} \times\left(\sigma^{2}+\sigma_{\text {ins }}^{2}\right)^{1 / 14} \\
e r r_{Z} & \approx 12 \times S N R^{-1} \times\left(\sigma^{2}+\sigma_{\text {ins }}^{2}\right)^{1 / 7}
\end{aligned}
$$

These relations are useful to select the observational setup: what is the optimal compromise between the spectral dispersion and the size of the spatial elements that minimizes the errors in a given observing time? Considering only the sources of uncertainties modeled in our simulations, it appears that the best precision on the internal kinematics will be obtained when $\sigma_{\text {ins }} \approx 0.8 \sigma$. For the best precision on the parameters of the stellar population it is preferable to maximize the total SNR with a lower dispersion. Still, a single setup with a resolution matching the velocity dispersion is in general the good choice.

The template mismatch due to abundance effects and the uncertainties in the modeling of a stellar populations are probably the source of significant biases on the parameters of the stellar population. In particular, we can guess that if Balmer lines are not in the wavelength range, the determination of age will be extremely sensitive to abundance mismatch. We have inverted observations of globular clusters [4] and simulated spectra from Bruzual \& Charlot [5]. For old populations the estimates appear too young and metallic. We are investigating the origin of this problem that may be connected with the bias found by Prieto et al. [6] in the ELODIE library.

\section{References}

1. Ocvirk et al., 2005, astro-ph/0507002

2. Chilingarian et al., 2005, Proceedings of IAUC198, p.105

3. Le Borgne et al., 2004, A\&A v.425, p.881

4. Rose et al., 2005, ApJS v.169, p.138

5. Bruzual \& Charlot, 2003, MNRAS v.344, p.1000

6. Prieto et al., astro-ph/0509812 
$S N R$ [per pixel, for $R=10000$, pixel $=10 \mathrm{~km} / \mathrm{s}$, range $=400-680 \mathrm{~nm}]$

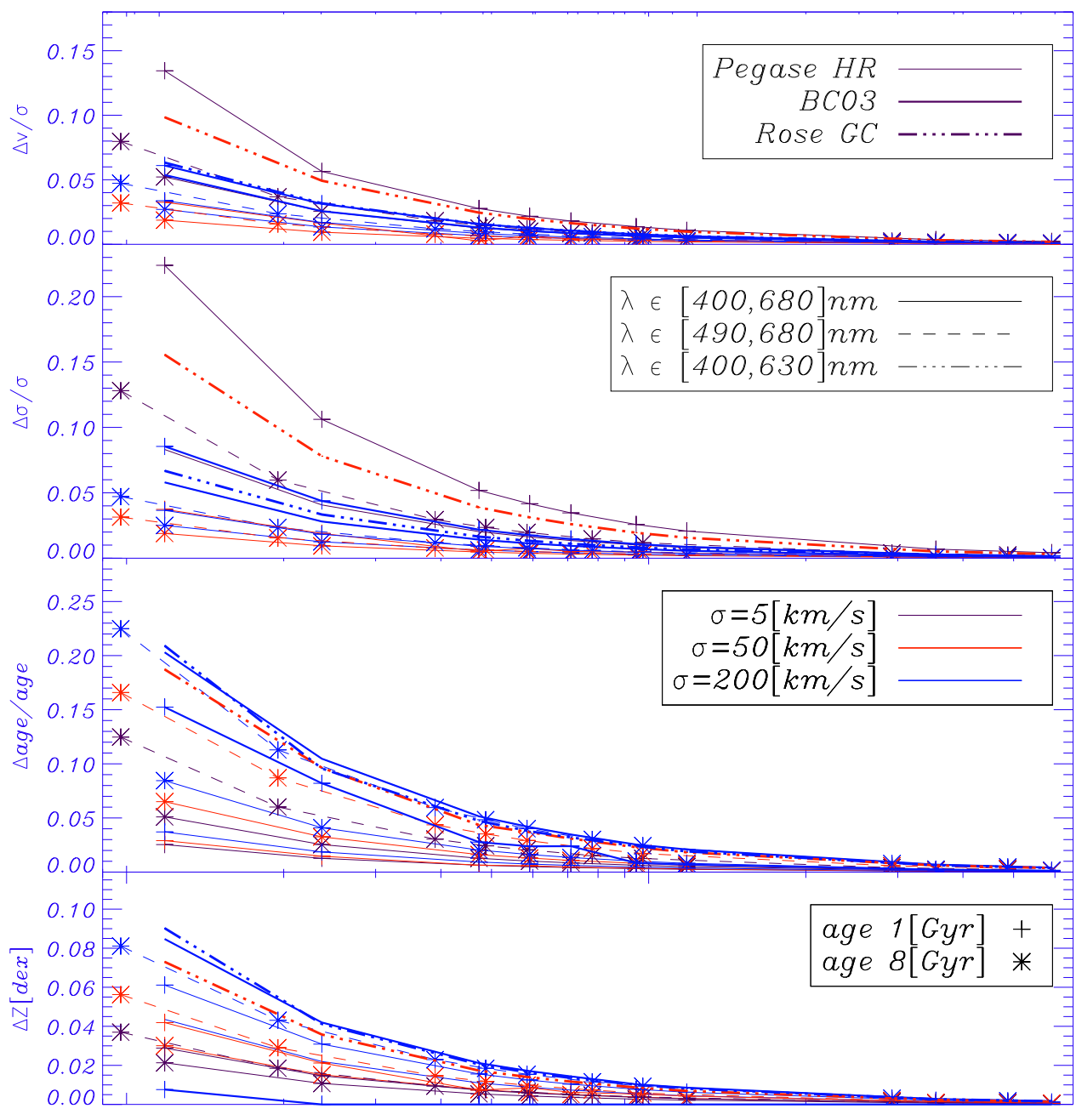

total SNR

Fig. 2. Error on population and kinematical parameters as a function of the total SNR. The upper $\mathrm{x}$ axis shows SNR per pixel for $\lambda$ range from 400 to $680 \mathrm{~nm}$ and dispersion of $0.2 \AA /$ pix. The curves noted 'BC03' are representing simulations with Bruzual \& Charlot (2003) models, and those noted 'Rose GC' are observation of globular clusters by Rose et al. (2005), all analyzed with PEGASE.HR templates. Errors are proportional to (total SNR) ${ }^{-1}$, see the formula in the text. 


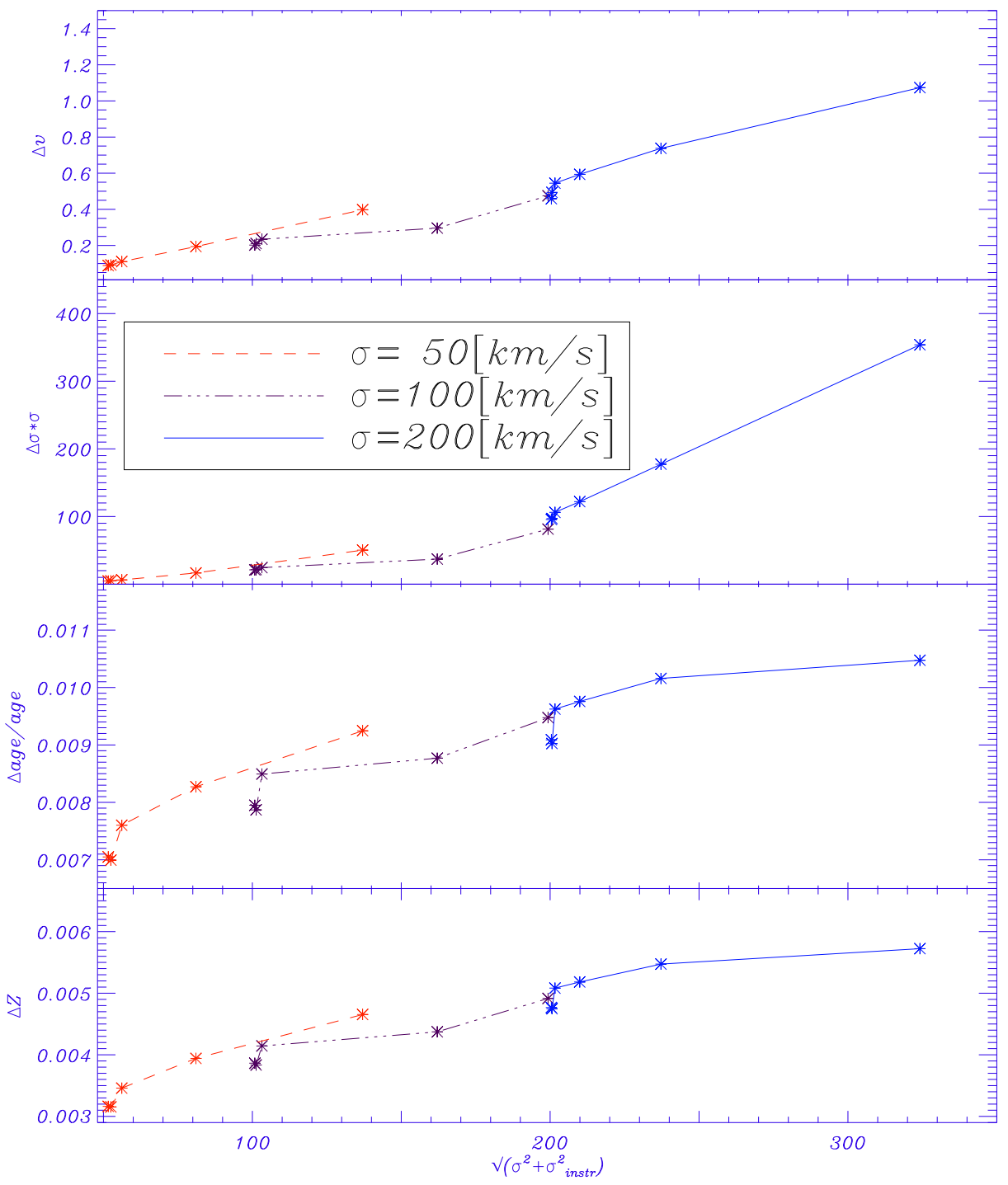

Fig. 3. Errors on the population and kinematical parameters as a function of the resolution. The instrumental dispersion ranges from $\sigma_{\text {ins }}=12 \mathrm{~km} / \mathrm{s}$ to $\sigma_{\text {ins }}=$ $250 \mathrm{~km} / \mathrm{s}(\mathrm{R}=500$ to 10000$)$. Errors are increasing with the effective broadening $\sqrt{\left(\sigma^{2}+\sigma_{\text {ins }}^{2}\right)}$ according to the formula given in the text. 